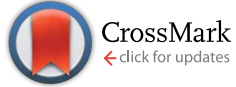

Cite this: RSC Adv., 2017, 7, 14290

Received 31st December 2016 Accepted 23rd February 2017

DOI: $10.1039 / c 6 r a 28868 e$

rsc.li/rsc-advances

\section{Tuning the surface chemistry of graphene flakes: new strategies for selective oxidation $\uparrow$}

\author{
Mariana P. Araújo, ${ }^{\text {a }}$ O. S. G. P. Soares, ${ }^{\text {b }}$ A. J. S. Fernandes, ${ }^{c}$ M. F. R. Pereira ${ }^{\text {b }}$ \\ and C. Freire*a
}

To accomplish a rational tuning of the surface chemistry of graphene flakes (GF), four different one-step protocols towards the selective oxidation of GF were performed, using different oxidants: nitric acid, potassium permanganate/sulfuric acid, ozone and 3-chloroperbenzoic acid. The characterization of the resulting materials confirmed the successful preparation of oxidized GF with $\mathrm{C} / \mathrm{O}$ atomic ratios varying in the range of 21.2-4.9, with distinct types of oxygen functionalities. While the oxidation of GF with nitric acid exclusively promotes the introduction of carboxylic groups and carbonyl/quinones, 3chloroperbenzoic acid is responsible for the introduction of epoxyl groups and carboxylic anhydrides, potassium permanganate favours the introduction of epoxyl and hydroxyl groups and some content of carboxylic anhydrides, and ozone promotes predominantly the introduction in graphene structure of epoxyl groups, carboxylic anhydrides, phenols, quinones and lactones, and in a lesser extension carbonyl groups in $\alpha$-substituted ketones and aldehydes if the oxidation is performed in the solid phase, or hydroxyl groups and a moderate content of carbonyl groups and aldehydes if GF are in a water dispersion. Furthermore, this work highlighted the possibility of identifying and distinguishing labile groups, namely hydroxyl and epoxyl groups, which are predominant in the structure of GF oxidized with potassium permanganate/sulfuric acid, ozone and 3-chloroperbenzoic acid. This is the first comprehensive study on the fine tuning of the surface of oxidized GF and a major contribution for the rational design of graphene composites since the application of these specific strategies can be useful in the anchoring of other molecules or nanoparticles.

\section{Introduction}

Graphene, an extended honeycomb network of $\mathrm{sp}^{2}$-hybridised carbon atoms and the first close-packed two-dimensional crystalline material isolated in nature, ${ }^{\mathbf{1 - 4}}$ has outstanding electronic, optical, thermal and mechanical properties such as ambipolar electric field effect, ${ }^{1}$ extremely high mobility of charge carriers, ${ }^{\mathbf{1 , 5}}$ high values of thermal conductivity, ${ }^{6}$ specific surface area, ${ }^{7}$ high chemical stability and high optical transmittance. ${ }^{7,8}$ Due to these remarkable properties, a great number of potential applications involving this nanomaterial have been

${ }^{a}$ REQUIMTE/LAQV, Departamento de Química e Bioquímica, Faculdade de Ciências, Universidade do Porto, Rua do Campo Alegre s/n, 4169-007 Porto, Portugal. E-mail: acfreire@fc.up.pt; Fax: +351 220402 695; Tel: +351 2204020590

${ }^{b}$ Laboratório de Catálise e Materiais (LCM), Laboratório Associado LSRE-LCM, Departamento de Engenharia Química, Faculdade de Engenharia, Universidade do Porto, Rua Dr Roberto Frias s/n, 4200-465 Porto, Portugal

'Instituto de Nanoestruturas, Nanomodelação e Nanofabricação (I3N), Departamento de Física, Universidade de Aveiro, Campus Universitário de Santiago, 3810-193 Aveiro, Portugal

$\uparrow$ Electronic supplementary information (ESI) available. See DOI: $10.1039 / \mathrm{c} 6 \mathrm{ra} 28868 \mathrm{e}$ observed and forecasted in several areas, such as electronics, energy conversion and storage or biotechnology.,

Despite the great potential applications of graphene, it possesses zero band gap and it is not very reactive. For most applications, graphene needs to be integrated with different functional materials; hence, the introduction of various chemical functionalities in graphene's backbone is mandatory in order to promote the formation of graphene composites with other nanomaterials and molecules. ${ }^{\mathbf{1 1}}$ Moreover, an obstacle to graphene processing and physical handling is its low dispersibility in both aqueous and organic solutions. Due to strong tendency to cluster together via $\pi-\pi$ stacking and van der Waals interactions, graphene sheets can form irreversible agglomerates or restack to form graphite. ${ }^{4}$ Thus, functionalization of graphene without damaging its electronic structure is crucial, in order to overcome its chemical inertness and to maximize its application.

In this context, most of the studies involve graphene oxide (GO), prepared through drastic oxidative conditions, or the material obtained upon its reduction (r-GO). ${ }^{2}$ Graphene oxide, which is commonly prepared via chemical oxidation of graphite and subsequent exfoliation of graphite oxide, has been exhaustively studied due to the possibility of immobilizing 
different nanomaterials onto GO surface, by taking advantage of its oxygen functionalities richness. ${ }^{12,13}$

Typically, graphene oxide has a carbon-oxygen ratio of 1.5 to 2.5 and can be produced by different chemical methods, being the most common the one proposed by Hummers and Offeman, ${ }^{14}$ which derived from the method of Staudenmaier, and the Brodie method..$^{15}$ According to the production processes and conditions, this material can have very distinct compositions and structures. ${ }^{16}$

The final structure of GO is highly dependent on differences in the starting materials (such as the graphite source) and the preparation methods, which complicates the exact identification and distribution of oxygen functional groups (usually, hydroxyls, epoxides, carbonyls, carboxylic acids, quinones and lactones) of the obtained structures. ${ }^{4,17}$ Currently, the knowledge of the exact bonding configuration and location of the oxygen chemical groups of GO is lacking, despite the fact that graphite oxide was firstly prepared 150 years ago. ${ }^{18}$ In the last decades, several possible graphite (and graphene) oxide models were suggested, based on several techniques such as nuclear magnetic resonance, X-ray diffraction, Fourier transform infrared spectroscopy, X-ray photoelectron spectroscopy and theoretical calculations, e.g. those proposed by Hofmann, Ruess, Scholz-Boehm and Nakajima-Matsuo, and more recently by Lerf-Klinowski, Dékány and Ajayan. ${ }^{18-24}$ Ruoff et al. reported for the first time the synthesis of a $100 \%{ }^{13} \mathrm{C}$-labelled graphite oxide for ${ }^{13} \mathrm{C}$ MAS NMR analysis. ${ }^{25}$ It highlighted some structural and spatial features: it was found that hydroxyl and epoxide carbons are bonded to each other, and carbonyl groups are separated from the most of the $\mathrm{sp}^{2}$, hydroxyl and epoxide groups. Furthermore, unattributed NMR signals were described, which Ajayan suggested to be due to the presence of lactols. $^{24}$

Although the high diversity of oxygen functionalities in GO is advantageous to promote the anchoring of nanoparticles or other chemical entities, its high degree of oxidation is responsible for considerable disruption of the electronic $\pi$ delocalization, even after reduction treatments, since the flow of charge carriers in reduced GO remains partially disrupted, severely affecting its electrochemical behaviour. ${ }^{26-28}$

Herein, the aim of the work was the selective oxidation of graphene flakes by four different one-step strategies in order to accomplish a rational tuning of the surface chemistry of graphene. In method A, nitric acid - one of the reagents employed in the Brodie method ${ }^{15}$ - was used as oxidant. In method B, graphene flakes were treated with potassium permanganate/ sulfuric acid, which are typically used in Hummers-Offeman method. ${ }^{14,29}$ In method C, the oxidation was promoted by ozone - to our knowledge, very few works have been reported concerning the modification of graphene-based materials with ozone $^{30-32}$ and most of the existing ones are focused on the graphene modification with ozone obtained through aggressive oxidation methods, such as the Hummers-Offeman method. ${ }^{30,31}$ Finally, in method D, graphene flakes were oxidized with 3-chloroperbenzoic acid (m-CPBA), which was only used in the epoxidation of carbon nanotubes ${ }^{33}$ and graphite. ${ }^{34}$ To achieve a full understanding on the effect on graphene structure, type of oxygen functional groups introduced during the oxidation protocols, composition, morphology and textural properties, a combination of several techniques were used. Finally, the electrochemical behaviour of the different oxidized graphene flakes was studied by cyclic voltammetry in the presence of $\left[\mathrm{Fe}(\mathrm{CN})_{6}\right]^{3-14-}$, in order to evaluate the influence of the oxygen surface groups on the electron transfer properties of oxidized graphene flakes. To the best of our knowledge, this is the first work on the fine tuning of graphene surface by selectively introducing distinct oxygen functional groups.

\section{Experimental}

\subsection{Reagents and solvents}

All the reagents and solvents used during the experimental work were used as received. Graphene flakes (GF) were commercially obtained from Graphene Technologies (Lot \#GTX-7/6-10.4.13). Nitric acid (>65\%, Merck), hydrogen peroxide solution $30 \mathrm{wt} \%$ in $\mathrm{H}_{2} \mathrm{O}$ (ACS reagent, Aldrich), potassium permanganate (ACS reagent, Merck), sulphuric acid (95-97\% Merck), 3-chloroperbenzoic acid (m-CPBA, $\leq 77 \%$ Aldrich), dichloromethane (99.99\% Fisher) and hydrochloric acid (37\% Fisher) were used in the oxidation of commercial GF. All the filtrations were performed through a polyamide membrane (Whatman, $45 \mu \mathrm{m}$ ). In the electrochemical studies, potassium hexacyanoferrate (III) (ACS reagent, Merck) and potassium chloride (99.999\% suprapur, Merck) were used and for the preparation of solutions Milli-Q water with a resistivity of $18.2 \mathrm{M} \Omega$ was used.

\subsection{Preparation of the oxidized graphene flakes}

Original graphene flakes (denoted as p-GF) were submitted to several oxidation treatments using different experimental conditions, after being sonicated for 10 minutes:

(i) Method A. Typically, $0.5 \mathrm{~g}$ of GF were refluxed in $\mathrm{HNO}_{3}$ $7 \mathrm{M}$ for 6 hours, at $90^{\circ} \mathrm{C}$; the resulting material was filtered and extensively washed with distilled water by reflux to neutral $\mathrm{pH}$. The material $\left(\mathrm{GF}_{-} \mathrm{HNO}_{3}\right)$ was dried under vacuum at $40{ }^{\circ} \mathrm{C}$.

(ii) Method B. A $9 \mathrm{M} \mathrm{H}_{2} \mathrm{SO}_{4}$ solution was slowly added to a mixture of $\mathrm{GF}(1.0 \mathrm{~g})$ and $\mathrm{KMnO}_{4}(1.0 \mathrm{~g})$, cooling the mixture in an ice bath at $0{ }^{\circ} \mathrm{C}$. The reaction was stirred for 6 hours, at room temperature, and then $450 \mathrm{~mL}$ of $\mathrm{H}_{2} \mathrm{O}$ and $2 \mathrm{~mL}$ of $\mathrm{H}_{2} \mathrm{O}_{2}(30 \%)$ were added. The mixture was filtered and the solid material was sequentially washed and centrifuged with $\mathrm{H}_{2} \mathrm{O}, \mathrm{HCl}(30 \%)$ and ethanol. The resulting $\mathrm{GF}\left(\mathrm{GF}_{-} \mathrm{KMnO}_{4}\right)$ was dried under vacuum at $40{ }^{\circ} \mathrm{C}$.

(iii) Method C. The ozonation of graphene flakes was obtained by bubbling $\mathrm{O}_{3}$ gas (produced from pure oxygen in a BMT $802 \mathrm{~N}$ ozone generator; $\mathrm{O}_{3}$ constant flowrate of $44.6 \mathrm{~g} \mathrm{~m}^{-3}$ ) through a graphene dispersion ( $1.0 \mathrm{~g}$ of GF in $350 \mathrm{~mL}$ of water) with stirring, for 5 hours (GF_O ${ }_{3}$ disp); subsequently, the material was filtered and dried under vacuum at $40{ }^{\circ} \mathrm{C}$. Another $\mathrm{O}_{3}$ treatment was carried out by passing the gas through $1.0 \mathrm{~g}$ of GF in the solid phase (GF_O ${ }_{3 \_}$gas).

(iv) Method D. Two experiments were performed in order to evaluate the m-CPBA concentration effect. $0.3 \mathrm{~g}$ of GF were added to a 0.1 or $0.01 \mathrm{M} \mathrm{m}$-CPBA solution in dichloromethane 
$(225 \mathrm{~mL})$; the mixtures were stirred for 6 hours, at $50{ }^{\circ} \mathrm{C}$. In both cases, after the reaction time, the mixtures were filtered and intensively washed with dichloromethane by reflux and dried in vacuum at $40{ }^{\circ} \mathrm{C}$. The resulting GF were denoted as GF_mCPBA_0.1 $\mathrm{M}$ and GF_m-CPBA_0.01 M.

\subsection{Physicochemical characterization}

X-ray photoelectron spectroscopy (XPS) was performed at Centro de Materiais da Universidade do Porto, Portugal (CEMUP), in a Kratos AXIS Ultra HSA spectrometer using monochromatized $\mathrm{Al} \mathrm{K} \alpha$ radiation $(1486.6 \mathrm{eV})$. The powdered materials were pressed into pellets prior to the XPS analysis. XPS spectra were deconvoluted with CasaXPS 2.3.12 software, using non-linear least squares fitting routine after a Shirley-type background subtraction and the peaks were interpreted using a combination of Gaussian/Lorentzian functions. The adjusted parameters were FWHM, binding energy and peak area. To correct possible deviations caused by electric charge of the samples, the C1s band at $284.6 \mathrm{eV}$ was taken as internal standard. Surface atomic percentages were calculated from the corresponding peak areas upon spectra deconvolution and using the sensitivity factors provided by the manufacturer.

Temperature programmed desorption (TPD) was performed in an AMI-200 apparatus, from Altamira Instruments. Typically, samples of $100 \mathrm{mg}$ were heated from room temperature up to $1100{ }^{\circ} \mathrm{C}$, at $5{ }^{\circ} \mathrm{C} \mathrm{min}{ }^{-1}$, under a flow of helium $\left(25 \mathrm{~cm}^{3} \mathrm{~min}^{-1}\right)$. The amounts of $\mathrm{CO}(\mathrm{m} / \mathrm{z}=28)$ and $\mathrm{CO}_{2}(\mathrm{~m} / \mathrm{z}=44)$ released from the samples were monitored with a Dymaxion mass spectrometer (Ametek Process Instruments). Spectra deconvolution was performed by using a multiple Gaussian function and the Levenberg-Marquardt iteration method.

Transmission electron microscopy (TEM) measurements were carried out at Instituto de Biologia Molecular e Celular (IBMC), Porto, Portugal. TEM micrographs were obtained with a JEOL JEM-1400 transmission electron microscope with a CCD camera Gatan, operating at $80 \mathrm{kV}$. Graphene samples were dispersed in ethanol (99.99\%, Fisher) under sonication, after which a carbon-coated 400 mesh copper grid was immersed in the suspension and then air-dried.

The micro-Raman analysis was conducted in the backscattering configuration on a Jobin Yvon HR800 instrument (Horiba, Japan), using a 1800 lines/mm grating and the $532 \mathrm{~nm}$ laser line from a Nd:YAG DPSS laser (Ventus, Laser Quantum, U.K.). For the Rayleigh rejection, a pair of edge filters placed in series were used allowing Raman acquisition from $50 \mathrm{~cm}^{-1}$. A $100 \times$ objective (spot size $\sim 2 \mu \mathrm{m}$, numeric aperture $=0.9$, Olympus, Japan) was used to focus the laser light onto the sample and to collect the backscattered Raman radiation to be detected by a Peltier cooled $(223 \mathrm{~K})$ CCD sensor. The spectrometer was operated in the confocal mode, setting the iris to $300 \mu \mathrm{m}$ and the acquisition time to $2 \times 10 \mathrm{~s}$. The data treatment of the acquired spectra (background removal and Gauss/ Lorentzian curve fitting) was carried out using the native NGS LabSpec software.

Fourier transform infrared spectroscopy (FTIR) spectra were obtained with a Jasco FT/IR-460 Plus spectrophotometer in the range of $400-4000 \mathrm{~cm}^{-1}$, with a resolution of $4 \mathrm{~cm}^{-1}$ and 32 scans. The spectra were collected in $\mathrm{KBr}$ pellets containing $0.2 \%$ weight of graphene materials.

The UV-visible spectra of graphene dispersions in water $(c=$ $0.1 \mathrm{mg} \mathrm{mL} \mathrm{mL}^{-1}$ ) were recorded at room temperature on a Shimadzu UV-2550 UV-vis spectrophotometer, in the range of 180$800 \mathrm{~nm}$, with a quartz cuvette (10 $\mathrm{mm}$ path length).

Electrochemical studies were carried out using an Autolab PGSTAT 30 potentiostat/galvanostat (controlled by GPES software). A three-electrode electrochemical cell was used with a glassy carbon (GC) electrode (3.0 $\mathrm{mm}$ of diameter, BASi) as working electrode, a Pt wire as counter electrode and an $\mathrm{Ag} / \mathrm{AgCl}$ $(1.0 \mathrm{M} \mathrm{NaCl})$ reference electrode (Metrohm). The reference electrode was separated from the working and counter electrodes through a porous glass frit and this compartment containing the reference electrode was filled with supporting electrolyte, which was a $0.1 \mathrm{M} \mathrm{KCl}$ solution. All the reported electrochemical potentials are referenced to the $\mathrm{Ag} / \mathrm{AgCl}$ reference electrode.

Cyclic voltammetry experiments in the presence of $1.0 \mathrm{mM}$ $\mathrm{K}_{3}\left[\mathrm{Fe}(\mathrm{CN})_{6}\right]$ solution were performed by cycling the potential 5 times between 0.9 and $-0.5 \mathrm{~V}$, at a scan rate of $0.1 \mathrm{~V} \mathrm{~s}^{-1}$. For the modification of GC electrode with graphene samples, GF, $\mathrm{GF}_{-} \mathrm{HNO}_{3}, \mathrm{GF} \_\mathrm{KMnO}_{4}, \mathrm{GF} \_\mathrm{O}_{3}$ disp, GF_O 3 _gas and GF_mCPBA were previously dispersed $\left(0.5 \mathrm{mg} \mathrm{mL}^{-1}\right)$ in DMF for 5 minutes with sonication. The immobilization onto the GC electrode surface was performed by drop-casting: $3 \mu \mathrm{l}$ of the corresponding material suspension were deposited with a micropipette and allowed to dry; prior to each material immobilization, the GC electrode surface was polished with alumina particles $(0.3 \mu \mathrm{m}$, Buehler $)$ and thoroughly washed with Milli-Q water. Electron transfer constants, $k_{\text {obs }}^{\circ}$, were determined according to the Nicholson approach, ${ }^{35}$ which establishes a relation between the $\Delta E_{\mathrm{p}-\mathrm{p}}$ values and the dimensionless kinetic parameter, $\psi$, and that recently has been applied to evaluate the heterogeneous electron transfer rates of graphene oxides. ${ }^{36,37}$ For the calculation of $k_{\text {obs }}^{\circ}$, diffusion coefficient values of $D_{\mathrm{o}}=7.39 \times 10^{-5}$ and $D_{\mathrm{r}}=6.81 \times 10^{-5} \mathrm{~cm}^{2} \mathrm{~s}^{-1}$ were used..$^{38}$

\section{Results and discussion}

\subsection{Composition, textural properties and morphology}

Surface composition data of original and oxidized graphene flakes ( $\mathrm{p}$ - and ox-GF, respectively) was obtained by XPS. The surface atomic percentages of each material are summarized in Table 1. Original GF (p-GF) is composed by $96.2 \%$ of carbon, $3.2 \%$ of oxygen and a residual amount of nitrogen $(0.6 \%)$. After the oxidation treatments, the amount of oxygen increased in all the materials, with the percentage varying between the lowest value of $4.5 \%$ for $\mathrm{GF}_{-} \mathrm{HNO}_{3}$ and the highest value of $16.8 \%$ for $\mathrm{GF}_{-} \mathrm{KMnO}_{4}$. C/O atomic ratios have considerably decreased, as a consequence of the oxygen percentage increase, for GF_KMnO 4 , GF_m-CPBA_0.1 M, GF_O $\mathrm{O}_{3}$ and GF_mCPBA_0.01 $\mathrm{M}$ (C/O ratios $=4.9,7.8,9.8$ and 13.0, respectively), when compared with the $\mathrm{C} / \mathrm{O}$ atomic ratio of $\mathrm{p}-\mathrm{GF}(\mathrm{C} / \mathrm{O}$ ratio $=$ 30.1). In the case of $\mathrm{GF}_{-} \mathrm{HNO}_{3}$, the XPS results confirmed the 
occurrence of a milder oxidation since the decrease of $\mathrm{C} / \mathrm{O}$ ratio is not so pronounced $(\mathrm{C} / \mathrm{O}$ ratio $=21.2)$. For comparison, $\mathrm{C} / \mathrm{O}$ atomic ratios of graphene oxides prepared by the Hummers method are in the range of 2.0-3.0 and, upon its reduction, $\mathrm{C} / \mathrm{O}$ ratios increase to $6.0-13.5,{ }^{17,27,39}$ which are close to the values obtained for the prepared ox-GF. In the case of graphene oxidation by ozone, $\mathrm{Z}$. Xu et al. were able to oxidize thermalexfoliated graphene nanosheets, achieving a $\mathrm{C} / \mathrm{O}$ ratio varying from 2.0 to 6.7 , although it is worth mentioning that the starting material had already considerably low $\mathrm{C} / \mathrm{O}$ ratio of 17.7 and thus the comparison should be done cautiously. ${ }^{30}$

The relative atomic percentages of carbon-based functional groups, which were determined based on the deconvolution of C1s high resolution spectra, are summarized in Table 2; deconvoluted C1s and O1s high resolution spectra of the most oxidized material, $\mathrm{GF}_{-} \mathrm{KMnO}_{4}$, are presented in Fig. 1, and deconvoluted C1s and O1s high resolution spectra of the other materials are presented in Fig. S1, ESI. $\dagger$

C1s high resolution spectra of all materials were deconvoluted into five peaks: a main peak at $284.6 \mathrm{eV}$ related to the graphitic structure $\left(\mathrm{sp}^{2}\right)$, a peak at $286.2 \mathrm{eV}$ attributed to $C-\mathrm{O}$ in hydroxyls, epoxy groups and phenols, a peak at $287.3 \mathrm{eV}$ associated to $C=\mathrm{O}$ in ketones, aldehydes and quinones, a peak at $288.9 \mathrm{eV}$, attributed to $\mathrm{O}-C=\mathrm{O}$ in carboxylic acids, carboxylic

Table 1 XPS surface atomic percentages and $\mathrm{C} / \mathrm{O}$ atomic ratios for the oxidized graphene flakes

\begin{tabular}{lllrr}
\hline & \multicolumn{2}{l}{$\%$} & \\
\cline { 2 - 3 } Graphene flakes & C1s & N1s & O1s & C/O ratio \\
\hline GF & 96.2 & 0.6 & 3.2 & 30.1 \\
GF_HNO & 95.5 & - & 4.5 & 21.2 \\
GF_O__disp $_{\text {GF_O__gas }}$ & 90.4 & 0.4 & 9.2 & 9.8 \\
GF_KMnO $_{4}$ & 90.3 & 0.5 & 9.2 & 9.8 \\
GF_m-CPBA 0.1 M & 82.3 & 0.9 & 16.8 & 4.9 \\
GF_m-CPBA 0.01 M & 88.3 & 0.4 & 11.3 & 7.8 \\
& 92.5 & 0.5 & 7.1 & 13.0
\end{tabular}

Table 2 Relative atomic percentages of carbon-based functional groups presented in the XPS high resolution C1s spectra

\begin{tabular}{|c|c|c|c|c|c|}
\hline \multirow[b]{2}{*}{$\begin{array}{l}\text { Graphene } \\
\text { flakes }\end{array}$} & \multicolumn{5}{|l|}{$\% \mathrm{C}$} \\
\hline & $\begin{array}{l}284.6 \mathrm{eV} \\
\text { (graphitic } \\
\text { structure) }\end{array}$ & $\begin{array}{l}286.3 \mathrm{eV} \\
\mathrm{C}-\mathrm{O}\end{array}$ & $\begin{array}{l}287.4 \mathrm{eV} \\
\mathrm{C}=\mathrm{O}\end{array}$ & $\begin{array}{l}288.9 \mathrm{eV} \\
\mathrm{COOH}\end{array}$ & $\begin{array}{l}290.3 \mathrm{eV} \\
\pi-\pi^{*}\end{array}$ \\
\hline GF & 71.4 & 5.8 & 5.4 & 1.9 & 15.5 \\
\hline $\mathrm{GF}_{1} \mathrm{HNO}_{3}$ & 79.8 & 4.4 & 4.4 & 2.6 & 8.8 \\
\hline GF_O ${ }_{3}$ disp & 76.4 & 6.4 & 5.3 & 0.5 & 11.4 \\
\hline GF_O 3 _gas & 67.6 & 13.8 & 6.6 & 1.1 & 10.8 \\
\hline $\mathrm{GF}_{-} \mathrm{KMnO}_{4}$ & 64.9 & 19.2 & 10.0 & 1.5 & 4.5 \\
\hline $\begin{array}{l}\text { GF_m-CPBA } \\
0.1 \mathrm{M}\end{array}$ & 76.8 & 6.3 & 4.9 & 0.4 & 11.6 \\
\hline $\begin{array}{l}\text { GF_m-CPBA } \\
0.01 \mathrm{M}\end{array}$ & 75.2 & 6.6 & 5.2 & 0.4 & 12.6 \\
\hline
\end{tabular}

from the $\mathrm{sp}^{2}$-hybrized carbon atoms at $290.3 \mathrm{eV} .^{\mathbf{4 0 - 4 5}}$

All spectra of the oxidized graphene flakes present a decrease of the relative intensity of the $\pi-\pi^{*}$ satellite peak, corroborating that the graphene oxidation originated a decrease in the electronic delocalization; this decrease is more accentuated for $\mathrm{GF}_{-} \mathrm{KMnO}_{4}$, which is the material that presented the highest degree of oxidation. Furthermore, the peaks attributed to carbon atoms bound to oxygen $(C-\mathrm{O}, C=\mathrm{O}, \mathrm{O}-C=\mathrm{O})$ of ox-GF show some variations in terms of intensity relatively to the corresponding p-GF peaks. For $\mathrm{GF}_{-} \mathrm{HNO}_{3}$, the relative atomic percentage of carbon attributed to $\mathrm{O}-\mathrm{C}=\mathrm{O}$ in carboxylic acids increased from 1.9 to $2.6 \%$. In the case of $\mathrm{GF}_{-} \mathrm{O}_{3}$ gas and $\mathrm{GF}_{-} \mathrm{KMnO}_{4}$, it is observed a considerable increase of the $C-\mathrm{O}$ peak (from 5.8 to 13.8 and $19.2 \%$ for p-GF, GF_O ${ }_{3}$ gas and $\mathrm{GF}_{\mathrm{K}} \mathrm{KMnO}_{4}$, respectively).

Fig. 2 shows the relative atomic percentage of oxygen for each contribution of O1s peak components; the corresponding deconvoluted O1s high resolution spectra and the relative atomic percentages of the oxygen-based functional groups are

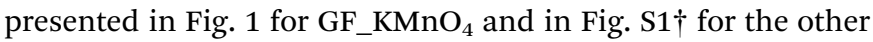
materials, and Table S1, ESI, $\uparrow$ respectively. For all materials, the broad band in the O1s high resolution spectra was deconvoluted into three peaks: ${ }^{\mathbf{4 6 , 4 7}}$ a peak at $531.3 \mathrm{eV}$, assigned to $O=\mathrm{C}$ in ketones, quinones and aldehydes, a peak at $532.7 \mathrm{eV}$, associated to $O-\mathrm{C}$ from phenol and epoxy groups, and a peak at $533.9 \mathrm{eV}$ typically due to $\mathrm{O}-\mathrm{C}=\mathrm{O}$ in esters and carboxylic acids.

The inspection of relative percentages of each type of oxygen groups, Fig. 2, shows for $\mathrm{GF}_{-} \mathrm{HNO}_{3}$ an increase in the percentage of the $\mathrm{O} 3$ component (attributed to $\mathrm{O}-\mathrm{C}=\mathrm{O}$ ), in comparison to $\mathrm{O} 1$ and $\mathrm{O} 2$ components, revealing the significant introduction of high acidic groups such as carboxylic acids, promoted by the oxidation treatment with $\mathrm{HNO}_{3}$.

In regard to $\mathrm{GF}_{-} \mathrm{O}_{3}$ gas and $\mathrm{GF}_{-} \mathrm{KMnO}_{4}$, the intensity of the peak at $532.7 \mathrm{eV}(\mathrm{O}-\mathrm{C})$ increased considerably relatively to the peak at $531.3 \mathrm{eV}(\mathrm{O}=\mathrm{C})$, when compared to the intensity of the corresponding peaks of p-GF (for GF_m-CPBA and GF_O $\mathrm{O}_{3}$ disp, the same trend is observed, although to a lesser extent), implying that functional groups such as epoxides, hydroxyls and phenols were introduced in a more extensive way than other functional groups such as ketones, quinones or aldehydes. These tendencies indicate that the type and the amount of oxygen groups introduced onto graphene surface are highly dependent on the type of oxidant; furthermore, the preparation form of graphene - dispersed in water or dried powdered during the oxidation with $\mathrm{O}_{3}$ also has some influence, since the relationship between the two peaks is quite different for $\mathrm{GF}_{-} \mathrm{O}_{3} \_$disp, in comparison with $\mathrm{GF}_{-} \mathrm{O}_{3}$ gas.

The content and the nature of oxygen functional groups were also assessed by TPD; it should be noted that, in the next sections, GF_m-CPBA refers to GF_m-CPBA_0.1 M. During a TPD analysis, all the oxygenated surface groups of carbon materials are thermally decomposed, releasing $\mathrm{CO}$ and/or $\mathrm{CO}_{2}$. carboxylic acids and lactones decomposition at low and high temperatures, respectively; carboxylic anhydrides originate both $\mathrm{CO}$ and $\mathrm{CO}_{2}$ at intermediate temperatures, and finally $\mathrm{CO}_{2}$ evolution spectrum results from the contribution of 

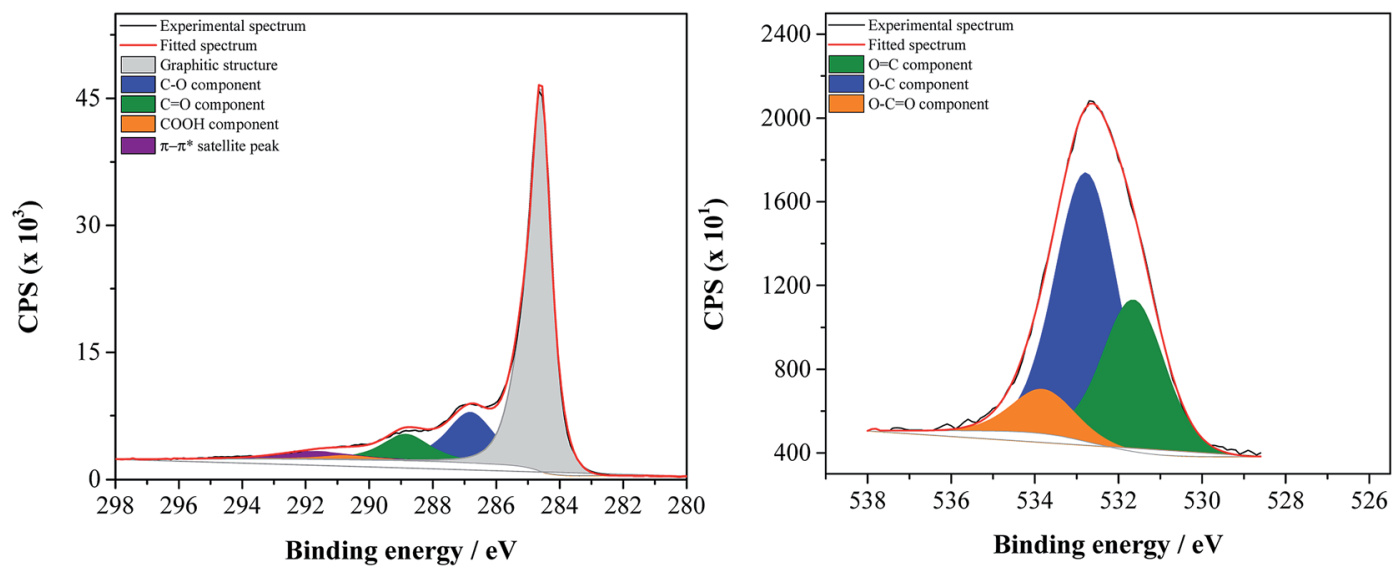

Fig. 1 Deconvoluted $\mathrm{C} 1 \mathrm{~s}$ (on the left) and $\mathrm{O} 1 \mathrm{~s}$ (on the right) high resolution spectra of $\mathrm{GF}_{2} \mathrm{KMnO}_{4}$.

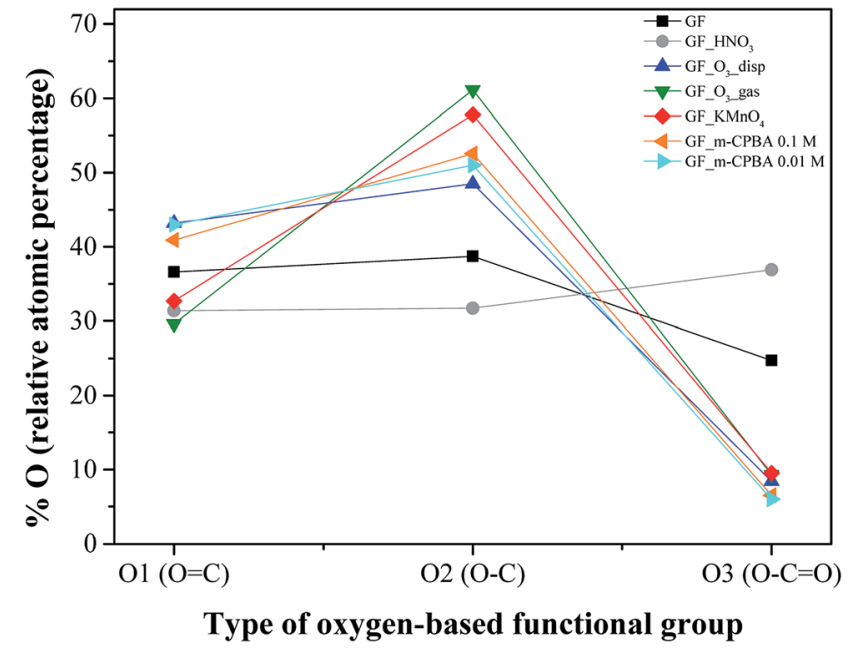

Fig. 2 XPS relative atomic percentage of oxygen for each contribution of O1s peak components: $\mathrm{O} 1, \mathrm{O} 2$ and $\mathrm{O} 3$ components correspond to oxygen in $\mathrm{O}=\mathrm{C}, \mathrm{O}-\mathrm{C}$ and $\mathrm{O}-\mathrm{C}=\mathrm{O}$, respectively.

phenols and carbonyl/quinone groups decompose in CO at high temperatures. ${ }^{\mathbf{4 8 , 4 9}}$

Fig. 3 shows the TPD spectra of p-GF and ox-GF materials. The amounts of $\mathrm{CO}_{2}$ and $\mathrm{CO}$ released obtained by integration of the areas under the respective TPD spectra, together with the total mass percentage of oxygen are presented in Table 3 . Globally, a substantial increase in the amounts of released $\mathrm{CO}_{2}$ and $\mathrm{CO}$ is observed for oxidized graphene flakes, as expected. For $\mathrm{GF}_{-} \mathrm{HNO}_{3}$, the overall profiles of $\mathrm{CO}_{2}$ and $\mathrm{CO}$ spectra are similar to the TPD profiles of p-GF, although the $\mathrm{CO}_{2}$ spectrum reveals an increase of $\mathrm{CO}_{2}$ evolution from 200 to $450{ }^{\circ} \mathrm{C}$, a temperature range ascribed to the decomposition of carboxylic acid groups, and from 450 to $650{ }^{\circ} \mathrm{C}$, which is indicative of the presence of carboxylic anhydrides, corroborating the XPS results that revealed a high proportion of carboxylic acids. In the case of $\mathrm{GF}_{-} \mathrm{KMnO}_{4}, \mathrm{GF} \_\mathrm{m}-\mathrm{CPBA}$ and $\mathrm{GF}_{-} \mathrm{O}_{3}$, there is a considerable increase in the amount of $\mathrm{CO}_{2}$ - specially for $\mathrm{GF}_{-} \mathrm{KMnO}_{4}$ and $\mathrm{GF} \_\mathrm{O}_{3}$ gas - indicating the introduction of carboxylic acid groups, carboxylic anhydrides and lactones $\left(\mathrm{CO}_{2}\right.$ released from 550 to $700{ }^{\circ} \mathrm{C}$ ). However, the TPD spectra of the previous oxidized GF present a distinctive feature - both $\mathrm{CO}_{2}$ and CO spectra present substantial peaks below $210{ }^{\circ} \mathrm{C}-$ that may be assigned to the decomposition of more labile groups, such as epoxyl or hydroxyl groups present in the basal plane, which is in agreement with TPD studies performed for graphite and graphene oxides by Dongil, ${ }^{50}$ Solís-Fernández ${ }^{51}$ and $\mathrm{Kuo}^{52}$ (although the precise differentiation between the temperature evolution of hydroxyl and epoxyl groups has not been yet clarified), and it is also corroborated by the work of Lerf, ${ }^{53}$ which demonstrated the elimination of epoxyl and hydroxyl groups upon calcination of graphite oxide, in vacuum at low temperature.

Deconvoluted $\mathrm{CO}_{2}$ and $\mathrm{CO}$ spectra are shown in Fig. S2, ESI $\dagger$ and the detailed information resulting from the deconvolution procedure, such as the temperature of the component peak maximum, $T_{\mathbf{M}}$, and the width at half-height, $W$, and the integrated area of each peak component, is presented in Tables 4 and 5. For TPD spectra deconvolution, the following assumptions were generally adopted: ${ }^{\mathbf{4 8 9}}$

(a) $\mathrm{CO}_{2}$ spectra results from the contribution of peak \#2, peak \#3 and peak \#4, attributed to carboxylic acids, carboxylic anhydrides and lactones, respectively; the same width at halfheight $(W$ ) was imposed for peaks \#2, \#3 and \#4;

(b) CO spectra are decomposed into four components: peak $\# 2$, attributed to the decomposition of carbonyl groups in $\alpha$ substituted ketones and aldehydes, and that has the same temperature of the component peak maximum $\left(T_{\mathbf{M}}\right)$ and $W$ as $\mathrm{CO}_{2}$ peak \#2; peak \#3 that results from the releasing of one $\mathrm{CO}$ molecule during carboxylic anhydride decomposition - this peak has the same $T_{\mathrm{M}}, W$ and magnitude as the $\mathrm{CO}_{2}$ peak \#3; peak \#4 and peak \#5, ascribed, respectively, to the decomposition of phenols and carbonyl/quinones - the $W$ was taken the same for both peaks.

(c) In this work, and for the specific cases of the samples $\mathrm{GF}_{-} \mathrm{KMnO}_{4}, \mathrm{GF} \_\mathrm{m}-\mathrm{CPBA}$ and $\mathrm{GF}_{-} \mathrm{O}_{3}$, an additional assumption was made: it was necessary to include a new $\mathrm{CO}$ and $\mathrm{CO}_{2}$ peak (peak \#1), at $T_{\mathrm{M}}<200{ }^{\circ} \mathrm{C}$. This peak is easily identified in both $\mathrm{CO}$ and $\mathrm{CO}_{2}$ spectra and with the same $T_{\mathrm{M}}$ and $W$. The only exception was for $\mathrm{GF}_{-} \mathrm{KMnO}_{4}$ sample, whose spectra clearly reveal the existence of two peaks in this region. 
a)

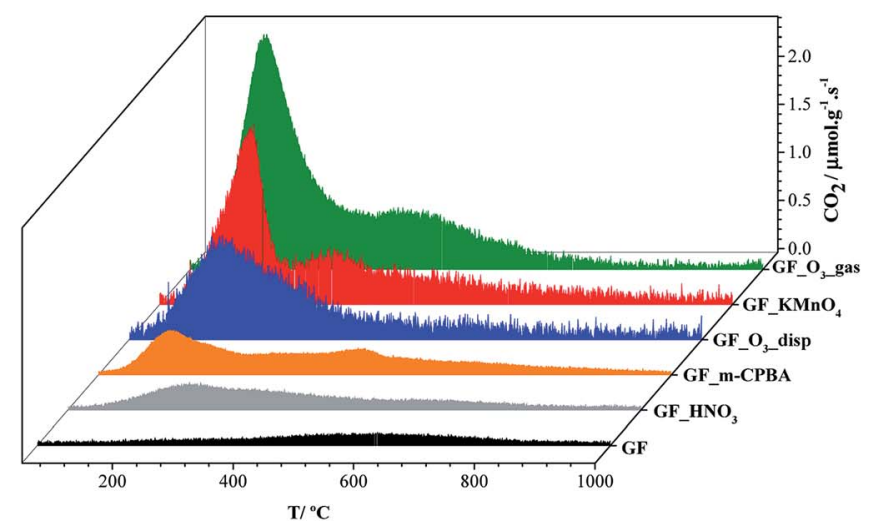

b)

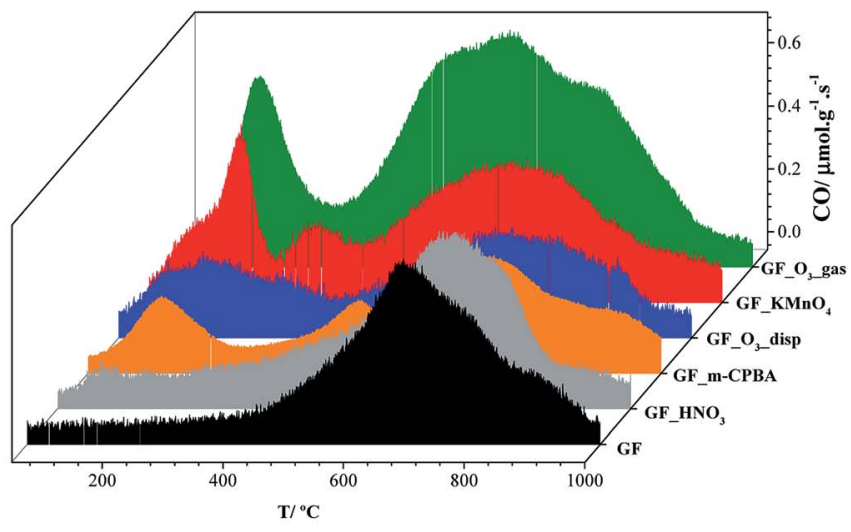

Fig. 3 TPD spectra of original and oxidized graphene flakes: (a) $\mathrm{CO}_{2}$ evolution and (b) $\mathrm{CO}$ evolution.

Table 3 Total amounts of $\mathrm{CO}_{2}$ and $\mathrm{CO}$ released by TPD and total mass percentage of oxygen obtained from TPD data (assuming that all the surface oxygen is released as $\mathrm{CO}$ and/or $\mathrm{CO}_{2}$ )

\begin{tabular}{lllr}
\hline Graphene flakes & {$\left[\mathrm{CO}_{2}\right] / \mu \mathrm{mol} \mathrm{g}^{-1}$} & {$[\mathrm{CO}] / \mu \mathrm{mol} \mathrm{g}^{-1}$} & $\% \mathrm{O}$ \\
\hline GF & 396 & 1608 & 3.8 \\
$\mathrm{GF}_{\text {GN_HO }}$ & 984 & 1692 & 5.9 \\
GF_KMnO $_{4}$ & 3036 & 2208 & 13.2 \\
GF_m-CPBA $_{\text {GF_O }}$ _disp & 1548 & 1836 & 7.9 \\
GF_O_gas & 2424 & 1524 & 10.2 \\
& 4872 & 4368 & 22.6
\end{tabular}

Regarding the region below $T=200{ }^{\circ} \mathrm{C}$, TPD spectra of GF_KMnO ${ }_{4}, \mathrm{GF} \_\mathrm{m}-\mathrm{CPBA}$ and $\mathrm{GF} \_\mathrm{O}_{3}$ gas present $\mathrm{CO}_{2}$ and $\mathrm{CO}$ peaks at $T=163-171{ }^{\circ} \mathrm{C}$, and $\mathrm{GF}_{-} \mathrm{O}_{3}$ disp spectra present a peak at $T=191{ }^{\circ} \mathrm{C}$, which is close to the temperature of the second fitted peak of $\mathrm{GF}_{-} \mathrm{KMnO}_{4}$ spectra $\left(T=203{ }^{\circ} \mathrm{C}\right)$. Based on our results, we ascribe the peaks at the lowest temperature, $T=$ 163-171 ${ }^{\circ} \mathrm{C}$, to the decomposition of epoxyl groups (which are unstable rings and therefore decompose at lower temperatures) and the peaks at $T=190-203{ }^{\circ} \mathrm{C}$ to the decomposition of hydroxyl groups. Thus, TPD spectra of $\mathrm{GF}_{-} \mathrm{KMnO}_{4}$ revealed that this material has an equivalent number of epoxyl and hydroxyl groups, since the first two peaks $\left(T=163{ }^{\circ} \mathrm{C}\right.$ and $\left.T=203{ }^{\circ} \mathrm{C}\right)$ correspond to the decomposition of epoxyl and hydroxyl groups and the relative percentages of each peak are similar. These results are in agreement with other reported works: according to the model of graphite oxide presented by Lerf et al. in $1988,{ }^{53}$ oxygen functionalities of GO prepared according to the Hummers method (and therefore using $\mathrm{KMnO}_{4}$ as one of the oxidizing agents) are mainly epoxyl and hydroxyl groups and Cai et al. have also demonstrated by ${ }^{13} \mathrm{C}$ solid-state NMR that each hydroxyl is usually accompanied by an epoxyl group. ${ }^{25,54}$ Moreover, Acik et al. have shown that during thermal annealing of graphene oxide, epoxyl groups are decomposed in the range of 60-175 ${ }^{\circ} \mathrm{C}$, while hydroxyl groups are only completely removed at higher temperatures, between 175 and $350{ }^{\circ} \mathrm{C} .{ }^{55}$

For graphene flakes treated with $0.1 \mathrm{M}$ m-CPBA solution, $\mathrm{CO}_{2}$ and $\mathrm{CO}$ peaks at $T=171^{\circ} \mathrm{C}$ corresponding to epoxyl species decomposition were also expected, since m-CPBA is typically used to promote epoxidation of $\mathrm{C}=\mathrm{C}$ in alkenes. ${ }^{33,34}$

Even though the results obtained by XPS indicate the occurrence of moderate oxidation for $\mathrm{GF}_{-} \mathrm{O}_{3}$ gas, in the TPD spectra this material exhibited the highest amount of global released of $\mathrm{CO}_{2}$ and $\mathrm{CO}$, comparable to the values observed for $\mathrm{GF}_{-} \mathrm{KMnO}_{4}$ (total amounts of $\mathrm{CO}_{2}$ and $\mathrm{CO}$ released correspond to 4872 and $4368 \mu \mathrm{mol} \mathrm{g}{ }^{-1}$, respectively, for $\mathrm{GF}_{-} \mathrm{O}_{3}$ gas, and 3036 and $2208 \mu \mathrm{mol} \mathrm{g}^{-1}$, respectively, for $\left.\mathrm{GF}_{-} \mathrm{KMnO}_{4}\right)$. The majority of the $\mathrm{CO}_{2}$ and $\mathrm{CO}$ evolution is observed at $T=171^{\circ} \mathrm{C}$, which can be assigned to epoxyl groups. According to a theoretical study performed by Lee, ozone gas molecules adsorb on graphene basal planes and epoxyl groups can be rapidly formed if the physisorbed molecule chemically react with graphene. ${ }^{56}$

Concerning GF_O ${ }_{3}$ disp, we assign the peak at $T=191{ }^{\circ} \mathrm{C}$ to the decomposition of hydroxyl groups. Although this material was prepared with ozone, the reaction occurred in the presence of water, which promotes the breakage of epoxyl groups to give two hydroxyl groups (per epoxyl). ${ }^{57}$ In sum, the analysis of the areas and the relative percentages of each peak of deconvoluted $\mathrm{CO}_{2}$ and $\mathrm{CO}$ spectra (Tables 4 and 5) allow us to reach some conclusions: (i) the oxidation of GF with $\mathrm{HNO}_{3}$ exclusively promotes the introduction of carboxylic groups and carbonyl/ quinones. Besides the carboxylic acids, the other oxidants also favour the formation of different oxygen functional groups: (ii) $\mathrm{m}$-CPBA is responsible for the introduction of epoxyl groups and carboxylic anhydrides; (iii) $\mathrm{GF}_{-} \mathrm{O}_{3}$ disp presents a high content of hydroxyl groups and a moderate content of carbonyl groups in $\alpha$-substituted ketones and aldehydes; (iv) GF_O ${ }_{3}$ gas presents predominantly in its structure epoxyl groups, carboxylic anhydrides, phenols, quinones and lactones, and in a lesser extension some carbonyl groups in $\alpha$-substituted ketones and aldehydes; (v) finally, the oxidation of GF with $\mathrm{KMnO}_{4}$ favours the introduction of epoxyl and hydroxyl groups and also some content of carboxylic anhydrides.

The morphology of ox_GF materials was analyzed by TEM and some examples of TEM micrographs of $\mathrm{p}$ - and ox-GF materials are shown in Fig. 4. The panel 4 a corresponds to a micrograph of original graphene flakes, while panels $4 \mathrm{~b}, 4 \mathrm{c}$ 


\begin{tabular}{|c|c|c|c|}
\hline & $\sum_{i}^{\mid} T_{0}$ & 字京车 & 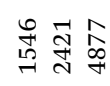 \\
\hline & 焉 & $m$ లె & 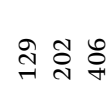 \\
\hline & 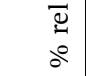 & 워 $\stackrel{\infty}{\sim}$ & 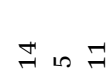 \\
\hline & 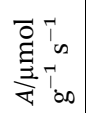 & 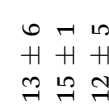 & 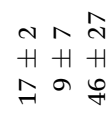 \\
\hline & $\hat{3}$ & 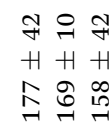 & 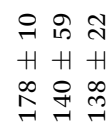 \\
\hline 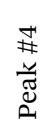 & $\frac{0}{0}$ & 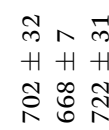 & 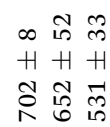 \\
\hline & $\begin{array}{c}\bar{D} \\
0\end{array}$ & \& $\stackrel{\infty}{\sim} \underset{\sim}{J}$ & $m a \stackrel{\infty}{\sim}$ \\
\hline & on & 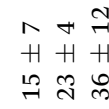 & 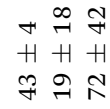 \\
\hline & $\overline{3}$ & 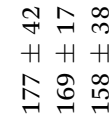 & 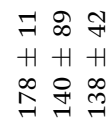 \\
\hline 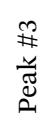 & $\frac{0}{0}$ & 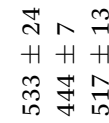 & 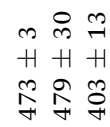 \\
\hline & 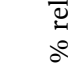 & 욤 & 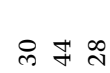 \\
\hline & 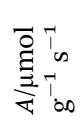 & 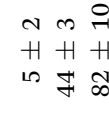 & 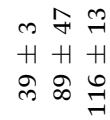 \\
\hline & $\frac{0}{3}$ & 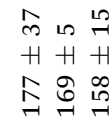 & 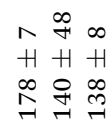 \\
\hline 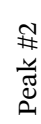 & $\sum_{i=1}^{0}$ & 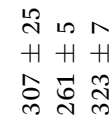 & 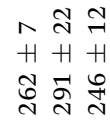 \\
\hline & $\begin{array}{c}\bar{D} \\
\circ \\
\end{array}$ & & $\ddot{2} \approx$ \\
\hline & 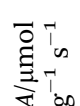 & & 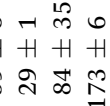 \\
\hline & 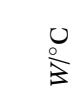 & & 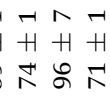 \\
\hline 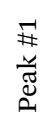 & तs & & 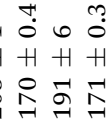 \\
\hline & 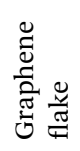 & 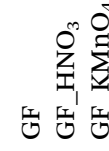 & 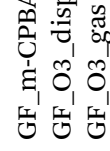 \\
\hline
\end{tabular}

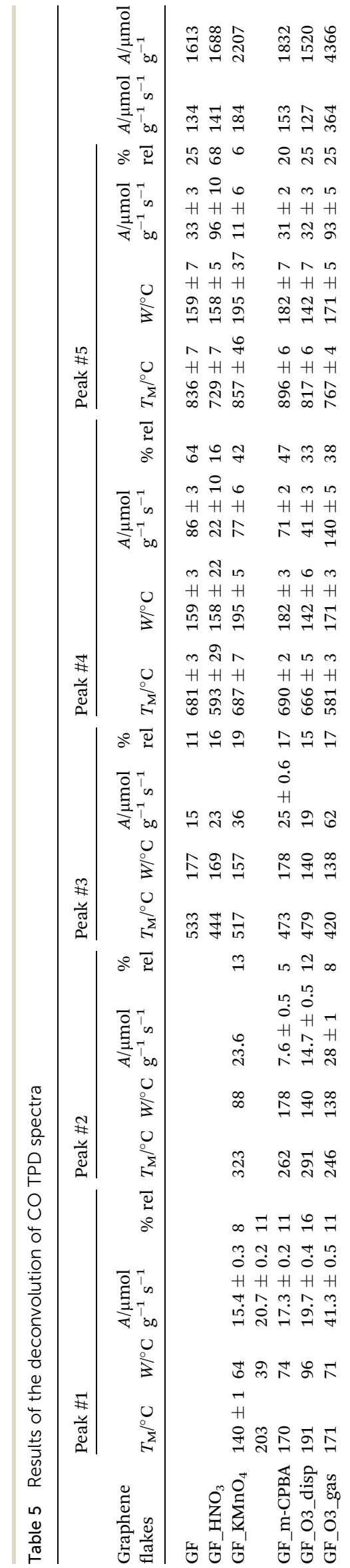


a)

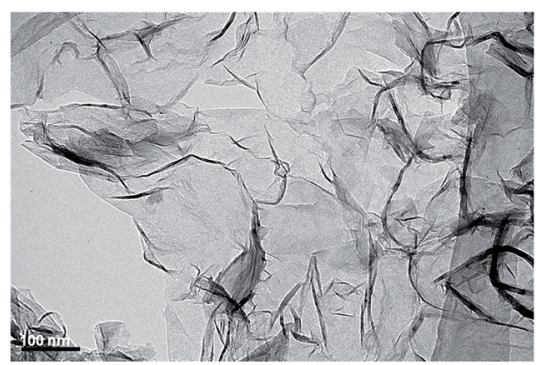

c)

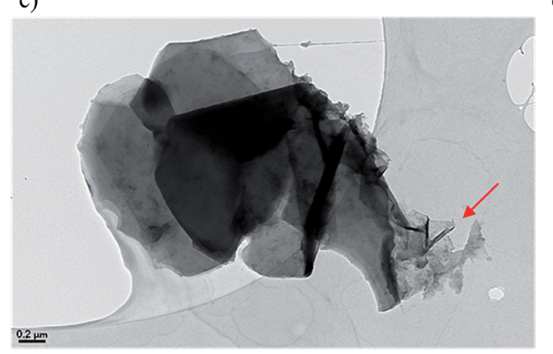

b)

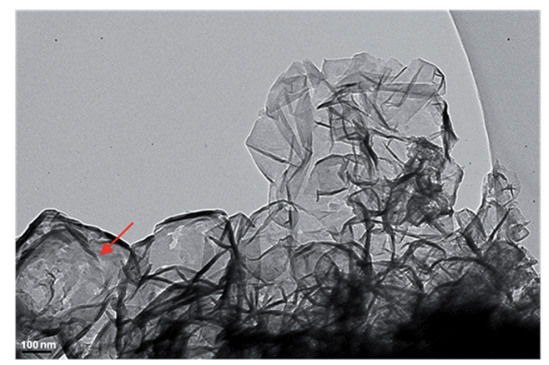

d)

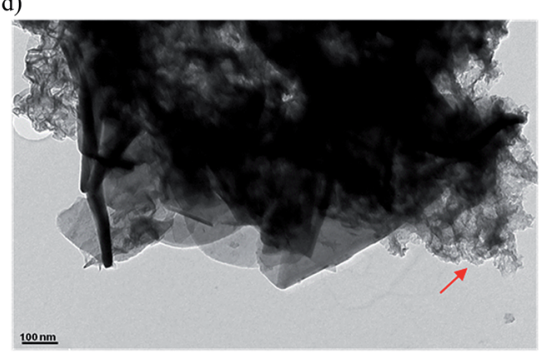

Fig. 4 TEM micrographs of (a) original and oxidized graphene flakes: (b) GF_HNO 3 ; (c) GF_KMnO 4 and (d) GF_O 3 _gas. Scale bars are 100 nm for $\mathrm{a}, \mathrm{b}$ and $\mathrm{d}$ and $0.2 \mu \mathrm{m}$ for $\mathrm{c}$. Defective regions are marked with a red arrow.

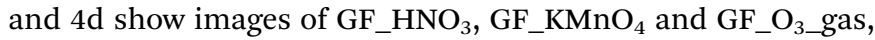
respectively. TEM images of the other oxidized graphene flakes are shown in Fig. S3, ESI. $\uparrow$ The acquired images suggest that p-GF is composed by wrinkled few-layer graphene sheets, whose sizes are in the range of hundreds of nanometres to one micrometre. In the case of the ox-GF - specially for $\mathrm{GF}_{-} \mathrm{KMnO}_{4}$ and $\mathrm{GF}_{-} \mathrm{O}_{3}$ gas - there are some regions where graphene sheets are defective (marked with a red arrow), probably due to the disorder induced by oxidation, coexisting with large and ordered flat sheets that are wrapped in some of the borders, as already reported. ${ }^{58-60}$ These wrapped sections may be caused by hydrogen bonding that may be formed between terminal groups, such as carboxyl, ketone and phenol groups within groups present in the basal planes, such as epoxy and hydroxyl groups. ${ }^{61}$

\subsection{Spectroscopic characterization}

Raman spectra of p- and ox-GF materials are shown in Fig. 5a. For all the materials, the corresponding spectrum displays a prominent $\mathrm{G}$ peak at $\sim 1584 \mathrm{~cm}^{-1}$, which is common to all $\mathrm{sp}^{2}$ carbon systems and corresponds to the first-order scattering of the $\mathrm{E}_{2 \mathrm{~g}}$ mode, and a peak at $\sim 1345 \mathrm{~cm}^{-1}$, ascribed to the $\mathrm{D}$ mode that is induced by structural disorder. In the second-order spectra of original and oxidized graphene flakes, there is a strong peak at $\sim 2700 \mathrm{~cm}^{-1}$, assigned as the $2 \mathrm{D}$ mode and that is the overtone of the D peak. At $\sim 1620 \mathrm{~cm}^{-1}$ it is also possible to identify a weak shoulder that corresponds to the $\mathrm{D}^{\prime}$ mode. The intensity ratio of the $\mathrm{D}$ and $\mathrm{G}$ bands is often used to estimate the disorder degree of graphitic materials. ${ }^{27,39,62,63}$ Fig. 5b presents the $I_{\mathrm{D}} / I_{\mathrm{G}}$ ratio for each material. Although the $I_{\mathrm{D}} / I_{\mathrm{G}}$
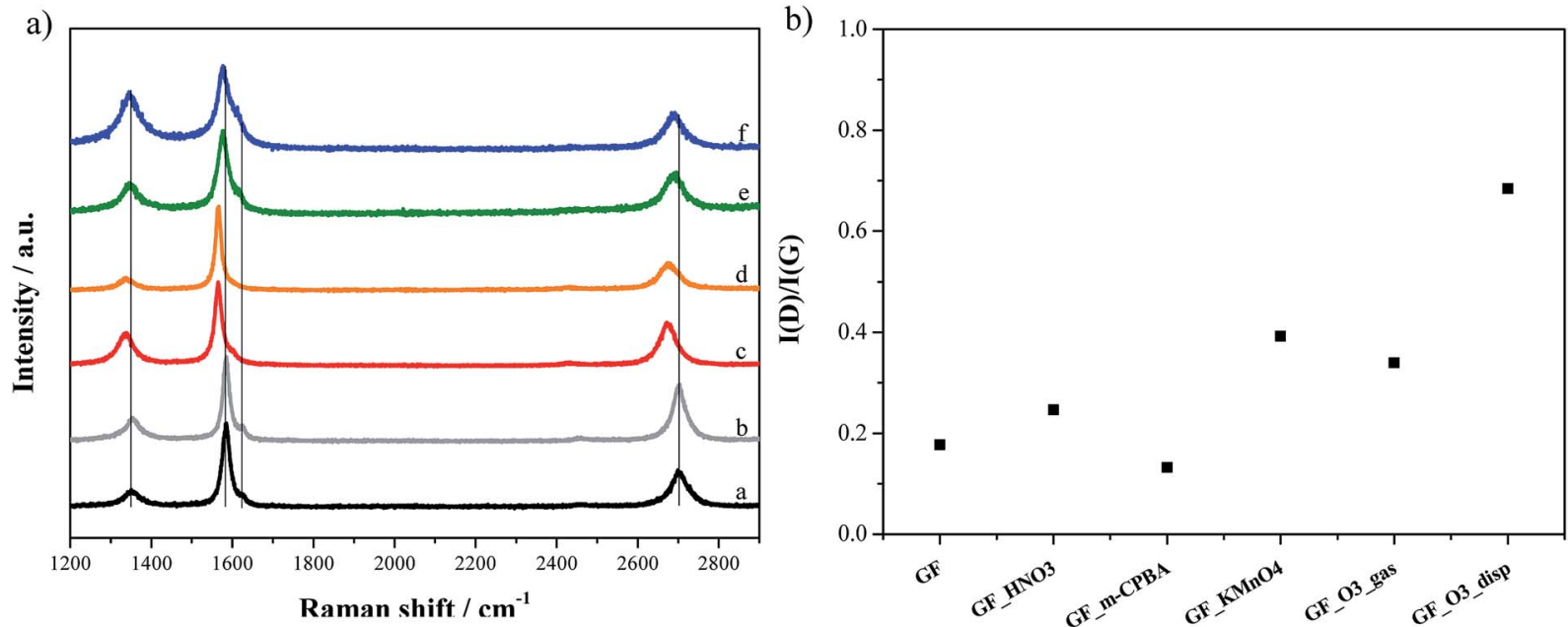

Fig. 5 (a) Raman spectra of original (a) and oxidized graphene flakes: GF_HNO 3 (b); GF_KMnO 4 (c); GF_m-CPBA (d); GF_O 3 _gas (e); GF_O 3 _disp (f). (b) Calculated $I_{D} / I_{G}$ ratio for original and oxidized graphene flakes. 
ratio remains practically constant for $\mathrm{GF}, \mathrm{GF} \_\mathrm{HNO}_{3}$ and $\mathrm{GF} \_\mathrm{m}$ CPBA, it increased considerably for $\mathrm{GF}_{-} \mathrm{O}_{3} \_$gas, $\mathrm{GF}_{-} \mathrm{KMnO}_{4}$ and $\mathrm{GF}_{-} \mathrm{O}_{3}$ disp, revealing the disorder effect of the introduction of oxygen functionalities on the structure of graphene. In fact, since the $I_{\mathrm{D}} / I_{\mathrm{G}}$ ratio is much higher, it can also reflect the occurrence of cracks and other structural defects during the oxidation treatment that originated this specific material.

The $I_{2 \mathrm{D}} / I_{\mathrm{G}}$ ratio is shown in Fig. S4, ESI. $\dagger$ The $2 \mathrm{D}$ mode is usually related to the crystalline structure and the stacking order, and also to the number of layers of graphene. ${ }^{6,65}$ The $I_{2 \mathrm{D}} /$ $I_{\mathrm{G}}$ ratios of $\mathrm{GF} \_\mathrm{HNO}_{3}$ and $\mathrm{GF}_{-} \mathrm{KMnO}_{4}$ materials are slightly higher than those of p-GF and the other oxidized materials; since $\mathrm{HNO}_{3}$ and $\mathrm{KMnO}_{4} / \mathrm{H}_{2} \mathrm{SO}_{4}$ are strong oxidants, an attack of the outer graphene layers may have occurred, leaving the oxidized graphene flakes only with the inner and more organized layers, and thus promoting an increase of the $I_{2 \mathrm{D}} / I_{\mathrm{G}}$ ratio.

Compared to the p-GF Raman spectrum, the oxidized graphene flakes, GF_O ${ }_{3}$ gas, GF_O $\mathrm{O}_{3}$ disp, GF_m-CPBA and $\mathrm{GF}_{-} \mathrm{KMnO}_{4}$, show the Raman bands shifted to lower frequencies, being this variation more accentuated for the last two materials. As observed in Fig. 6, the shift is larger for 2D band. This shift can be caused by the existence of tension in the structure of graphene, whose origin may be related to the introduction of epoxyl and hydroxyl groups onto the basal planes of graphene. Furthermore, Ruoff et al. had firstly observed a similar relationship between the degree of oxidation of graphene oxide films and the variation of $2 \mathrm{D}$ peak position. ${ }^{\mathbf{6 6}}$

FTIR spectra were also obtained to confirm the presence of oxygen functional groups in the ox-GF materials, Fig. 7. The FTIR spectrum of p-GF shows two bands: a band at $1580 \mathrm{~cm}^{-1}$, assigned to the $\mathrm{C}=\mathrm{C}$ stretching mode of skeletal vibrations from non-oxidized domains, and a broad band at $1220 \mathrm{~cm}^{-1}$ that results from the contribution of phenolic groups vibration modes. ${ }^{31}$ The FTIR spectra of the oxidized graphene flakes present more resolved vibration bands, since the oxidation introduces some disruption of the electronic conjugation and

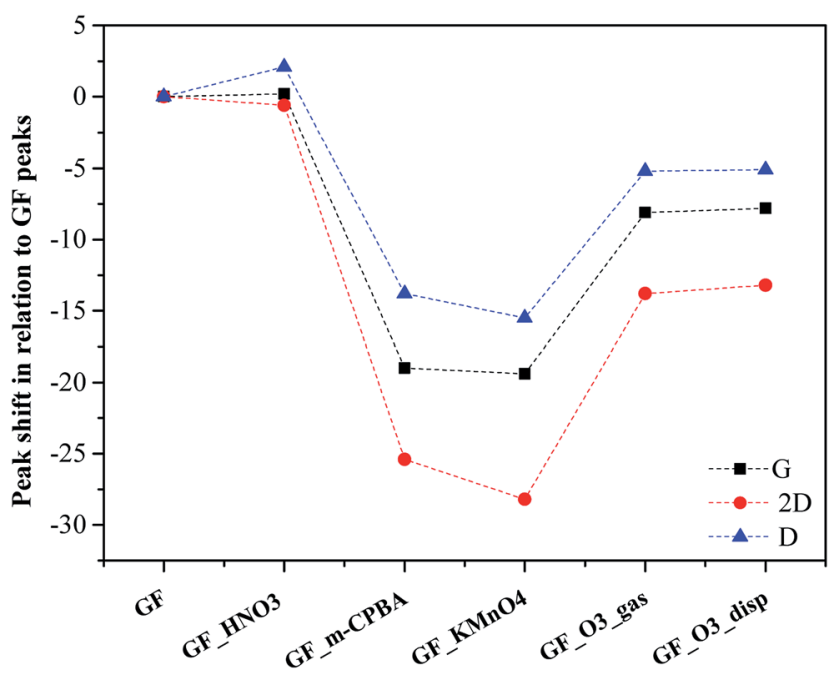

Fig. 6 Raman shift of D, G and 2D peak positions for oxidized graphene flakes, in relation to the Raman shift of the corresponding peaks of original graphene flakes.

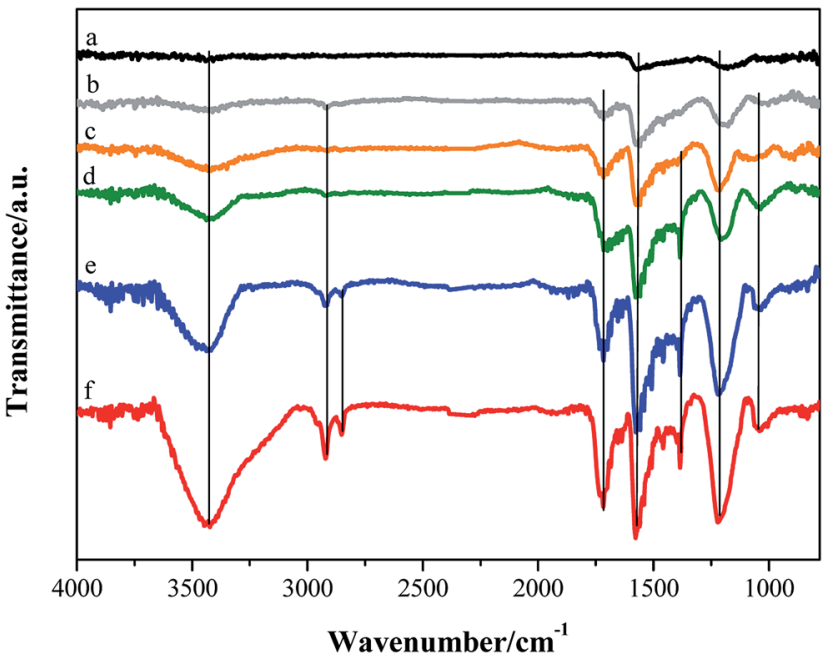

Fig. 7 FTIR spectra of (a) original and oxidized graphene flakes: (b) GF_HNO ${ }_{3}$; (c) GF_m-CPBA; (d) GF_O3_gas; (e) GF_O3_disp; (f) $\mathrm{GF}_{-} \mathrm{KMnO}_{4}$.

leads to the rupture of some $\pi-\pi$ interactions. Besides the two vibrational bands stated above, these spectra also show typical vibrational bands of $\mathrm{OH}$ groups at $3430 \mathrm{~cm}^{-1}$ and $1340 \mathrm{~cm}^{-1}$, due to $\mathrm{O}-\mathrm{H}$ stretching and bending modes, ${ }^{31,67}$ respectively. The spectra of GF_KMnO ${ }_{4}$ and $\mathrm{GF}_{-} \mathrm{O}_{3}$ disp materials present two bands at $2935 \mathrm{~cm}^{-1}$ and $2850 \mathrm{~cm}^{-1}$, assigned - respectively - to the $\mathrm{C}-\mathrm{H}$ bending and stretching modes that can be due to the existence of defects in the $\mathrm{sp}^{2}$ hybridized domains. The FTIR spectra of all ox-GF materials also reveal a vibrational band at $1740 \mathrm{~cm}^{-1}$ due to the $\mathrm{C}=\mathrm{O}$ stretching mode of carboxylic acid, carbonyl, ketone and quinone groups. ${ }^{68,69}$ It is also worth mentioning that the band at $1220 \mathrm{~cm}^{-1}$ is considerably more intense in the spectra of the oxidized graphene flakes, when compared with the band of the parent material, which suggests the introduction of phenol/hydroxyl groups. A broad and

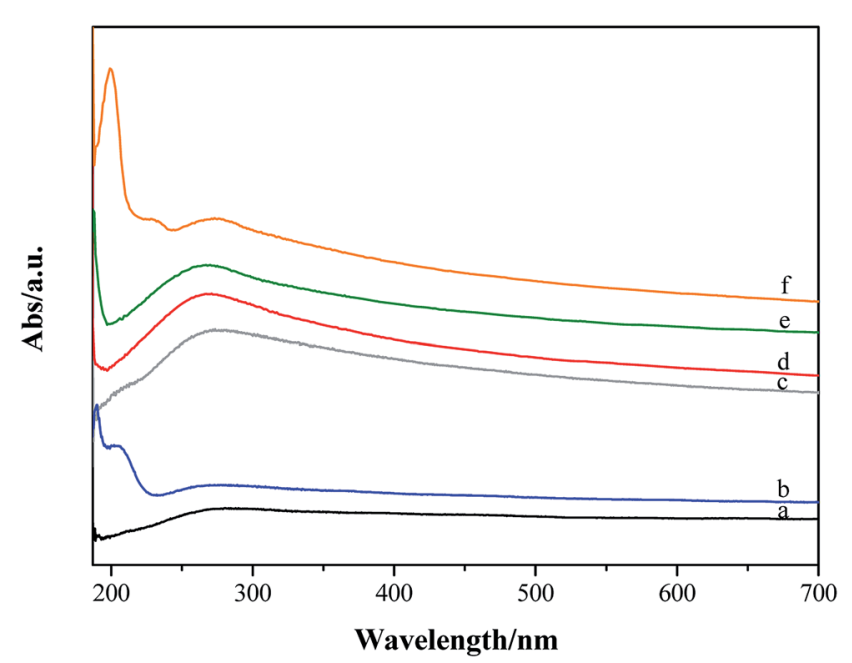

Fig. 8 UV-visible absorption spectra of (a) original and oxidized graphene flakes: (b) GF_O 3 disp; (c) $\mathrm{GF}_{-} \mathrm{HNO}_{3}$; (d) $\mathrm{GF}_{-} \mathrm{KMnO}_{4}$; (e) GF_O3_gas; (f) GF_m-CPBA. 
a)

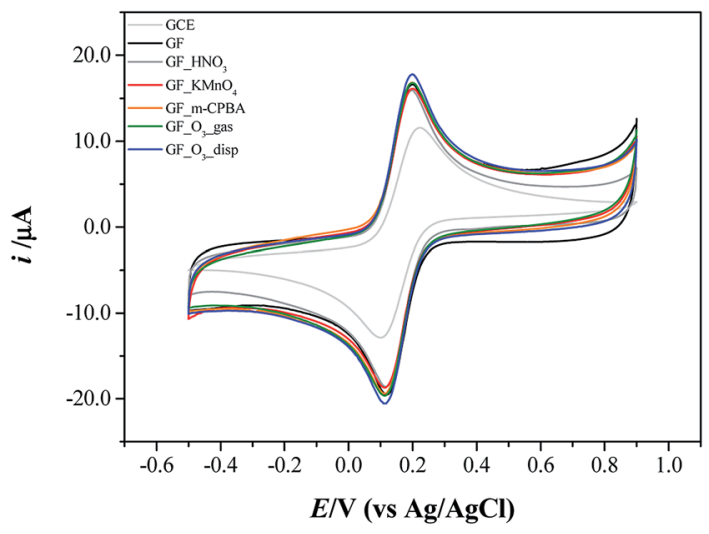

b)

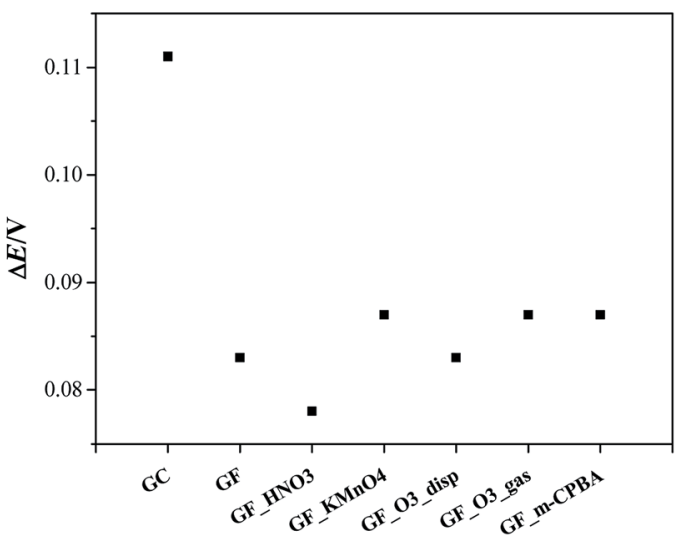

Fig. 9 (a) Cyclic voltammetry of original and oxidized graphene flakes-modified glassy carbon (GC) electrode in $1.0 \mathrm{mM} \mathrm{K} \mathrm{K}_{3}\left[\mathrm{Fe}(\mathrm{CN})_{6}\right]$ solution $\left(0.1 \mathrm{M} \mathrm{KCl}\right.$ as supporting electrolyte); (b) peak-to-peak separation of $\left[\mathrm{Fe}(\mathrm{CN})_{6}\right]^{3-/ 4-}$ redox couple for original and oxidized graphene flakes modified-glassy carbon (GC) electrode.

prominent band at $1050 \mathrm{~cm}^{-1}$ also appears in the FTIR spectra

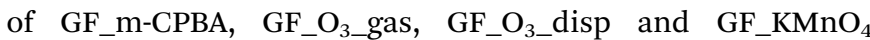
materials that can be assigned to $\mathrm{C}-\mathrm{O}-\mathrm{C}$ and $\mathrm{C}-\mathrm{O}$ stretching modes and $\mathrm{C}-\mathrm{O}-\mathrm{H}$ bending mode (in this spectral region typically occurs the overlap of several $\mathrm{C}-\mathrm{O}$ vibration modes, ${ }^{70}$ and thus the attribution of $\mathrm{C}-\mathrm{O}$ and $\mathrm{C}-\mathrm{OH}$ is still ambiguous $^{31,68,69,71,72}$ ).

UV-visible absorption spectra of p-GF and ox-GF materials are shown in Fig. 8. When compared with the spectrum of p-GF, the spectra of ox-GF present a prominent peak at $\lambda \sim 270$ $280 \mathrm{~nm}$ that can be assigned to $\mathrm{n} \rightarrow \pi^{*}$ transition of $\mathrm{C}=\mathrm{O}$ bonds. ${ }^{73}$ The intensity of this band is higher for $\mathrm{GF}_{-} \mathrm{KMnO}_{4}$ and $\mathrm{GF}_{-} \mathrm{O}_{3 \_}$gas, which is indicative of a higher degree of oxidation and, hence, corroborates our previous results. For GF_m-CPBA, an additional band at $\lambda=200 \mathrm{~nm}$ and a shoulder at $\lambda=230 \mathrm{~nm}$ is also observed; in addition, the spectrum of $\mathrm{GF}_{-} \mathrm{O}_{3}$ disp shows a band at $\lambda=210 \mathrm{~nm}$, the same region in which there is a shoulder in the spectrum of $\mathrm{GF}_{-} \mathrm{HNO}_{3}$. No assignment could be made to these observed bands.

\subsection{Electrochemical characterization}

Voltammetric experiments in the presence of the $\left[\mathrm{Fe}(\mathrm{CN})_{6}\right]^{3-/ 4-}$ redox probe were performed. This is a standard probe which has been used to assess the influence of surface oxygen and other functional groups of several carbon materials, such as pyrolytic graphite, carbon nanotubes and graphene oxide, on the electrode kinetics..$^{37,74,75}$

Cyclic voltammograms of bare and modified glassy carbon electrode with original and oxidized graphene flakes in $1.0 \mathrm{mM}$ $\mathrm{K}_{3}\left[\mathrm{Fe}(\mathrm{CN})_{6}\right]$ solution $(0.1 \mathrm{M} \mathrm{KCl}$ as supporting electrolyte $)$ are shown in Fig. 9a. The cyclic voltammogram of the bare electrode shows the oxidation and the reduction peaks of $\left[\mathrm{Fe}(\mathrm{CN})_{6}\right]^{3-/ 4-}$ at $E_{\mathrm{pa}}=0.216 \mathrm{~V}$ and $E_{\mathrm{pc}}=0.105 \mathrm{~V}$, respectively. In the CVs of the $\mathrm{p}$ - and ox-GF-modified electrodes, the intensity of the oxidation and reduction peaks ( $i_{\mathrm{pa}}$ and $i_{\mathrm{pc}}$, respectively) is higher than the intensity of the correspondent peaks for the bare GC electrode. Furthermore, the modification of the GC electrode surface with original and oxidized graphene flakes results in a decrease of peak-to-peak separation $\left(\Delta E_{\mathrm{p}-\mathrm{p}}\right)$, as it is presented in Fig. $9 \mathrm{~b}$. Consequently, the decrease of the $\Delta E_{\mathrm{p}-\mathrm{p}}$ is indicative of a faster electron transfer that can be quantified by the faster electron transfer constant, $k_{\mathrm{obs}}^{\circ}$ : the $k_{\mathrm{obs}}^{\circ}$ values, determined by the Nicholson methodology, for bare GC electrode is $4.26 \times 10^{-5} \mathrm{~cm} \mathrm{~s}^{-1}$, while the $k_{\text {obs }}^{\circ}$ values for GF, GF_HNO ${ }_{3}, \mathrm{GF}_{-} \mathrm{O}_{3}$ disp are respectively: $1.04 \times 10^{-4}, 1.37 \times 10^{-4}$ and $1.04 \times 10^{-4} \mathrm{~cm} \mathrm{~s}^{-1}$, and for GF_m-CPBA, GF_KMnO ${ }_{4}$, and GF_O ${ }_{3}$ gas is $8.74 \times 10^{-5} \mathrm{~cm} \mathrm{~s}^{-1}$.

Interestingly, the modification of the $\mathrm{GC}$ electrode with original and oxidized graphene flakes leads to similar results. The existence of oxygen functional groups in graphene can be responsible for the decrease of the electron transfer of $\left[\mathrm{Fe}(\mathrm{CN})_{6}\right]^{3-/ 4-}$ redox couple due to electrostatic repulsion between $\left[\mathrm{Fe}(\mathrm{CN})_{6}\right]^{3-/ 4-}$ and the functionalized graphene surface ${ }^{76}$ so the same trend was not expected for both original and oxidized graphene flakes. Hence, the obtained results suggest that the applied mild oxidation protocols do not affect the electrochemical behaviour of original graphene flakes.

\section{Conclusions}

The selective oxidation of graphene flakes was successfully achieved through the implementation of four different one-step protocols based on distinct oxidative systems. XPS results confirmed the occurrence of graphene oxidation in different degrees, with $\mathrm{C} / \mathrm{O}$ atomic ratios ranging from 21.2 to 4.9. TPD and FTIR characterization also revealed that it was possible to tune the surface chemistry of graphene flakes, according to the type of oxidant: the introduction of carboxylic acid groups was predominant in the case of the oxidation reaction with $\mathrm{HNO}_{3}$, while more labile groups such as epoxyl and hydroxyl groups could be introduced in the basal planes when the oxidations were performed with $\mathrm{KMnO}_{4}, \mathrm{~m}-\mathrm{CPBA}$ and $\mathrm{O}_{3}$. Besides, the oxidation with $\mathrm{O}_{3}$ revealed that the initial state of graphene flakes is determinant since $\mathrm{GF}_{-} \mathrm{O}_{3}$ disp presented a high amount of hydroxyl groups and a moderate content of carbonyl 
groups in $\alpha$-substituted ketones and aldehydes, while $\mathrm{GF}_{-} \mathrm{O}_{3-}$ gas presented predominantly in its structure epoxyl groups, carboxylic anhydrides, phenols, quinones and lactones, and in a lesser extension carbonyl groups in $\alpha$-substituted ketones and aldehydes. Moreover, this work highlighted the potentiality of TPD analysis in distinguishing epoxyl and hydroxyl groups on graphene flakes surface.

The selective one-step oxidation strategies presented in this work contribute to a step forward in the rational design of graphene-based composites with potential applications in electronics and energy storage and conversion, without compromising the disruption of the electronic $\pi$ delocalization of graphene and avoiding multiple, exhaustive and drastic steps involved in the preparation of GO and rGO based materials.

\section{Acknowledgements}

The work was funded by Fundação para a Ciência e a Tecnologia (FCT)/MEC under FEDER under Program PT2020 (projects UID/ QUI/50006/2013-POCI/01/0145/FEDER/007265 and UID/EQU/ 50020/2013-POCI-01-0145-FEDER-006984) and Program FCT UT Austin (project ref UTAP-ICDT/CTM-NAN/0025/2014). MA thanks FCT for her PhD grant (SFRH/BD/89156/2012) and support from Fundação Calouste Gulbenkian through Programa de Estímulo à Investigação 2013, OSGPS thanks FCT for the BPD grant (SFRH/BPD/97689/2013).

\section{References}

1 K. S. Novoselov, A. K. Geim, S. V. Morozov, D. Jiang, Y. Zhang, S. V. Dubonos, I. V. Grigorieva and A. A. Firsov, Science, 2004, 306, 666-669.

2 M. Inagaki, Y. A. Kim and M. Endo, J. Mater. Chem., 2011, 21, 3280-3294.

3 R. Mas-Ballesté, C. Gómez-Navarro, J. Gómez-Herrero and F. Zamora, Nanoscale, 2011, 3, 20-30.

4 Q. Tang, Z. Zhou and Z. Chen, Nanoscale, 2013, 5, 4541-4583. 5 A. K. Geim, Science, 2009, 324, 1530-1534.

6 A. A. Balandin, S. Ghosh, W. Bao, I. Calizo, D. Teweldebrhan, F. Miao and C. N. Lau, Nano Lett., 2008, 8, 902-907.

7 C. N. R. Rao, A. K. Sood, K. S. Subrahmanyam and A. Govindaraj, Angew. Chem., Int. Ed., 2009, 48, 7752-7777.

8 S. Guo and S. Dong, Chem. Soc. Rev., 2011, 40, 2644-2672.

9 N. Yang, G. M. Swain and X. Jiang, Electroanalysis, 2015, 2734.

10 D. Higgins, P. Zamani, A. Yu and Z. Chen, Energy Environ. Sci., 2016, 9, 357-390.

11 H.-X. Wang, Q. Wang, K.-G. Zhou and H.-L. Zhang, Small, 2013, 9, 1266-1283.

12 C. K. Chua and M. Pumera, Chem. Soc. Rev., 2014, 43, 291312.

13 X. An and J. C. Yu, RSC Adv., 2011, 1, 1426-1434.

14 W. S. Hummers and R. E. Offeman, J. Am. Chem. Soc., 1957, 208, 1939.

15 B. C. Brodie, Ann. Chim. Phys., 1860, 59, 466-472.

16 J.-A. Yan and M. Y. Chou, Phys. Rev. B: Condens. Matter Mater. Phys., 2010, 82, 125403-125410.
17 D. R. Dreyer, S. Park, C. W. Bielawski and R. S. Ruoff, Chem. Soc. Rev., 2010, 39, 228-240.

18 T. Szabó, O. Berkesi, P. Forgó, K. Josepovits, Y. Sanakis, D. Petridis and I. Dékány, Chem. Mater., 2006, 18, 2740-2749.

19 U. Hofmann and R. Holst, Ber. Dtsch. Chem. Ges., 1939, 72, 754-771.

20 G. Reuss, Monatsh. Chem., 1946, 76, 381-417.

21 W. Scholz and H. P. Boehm, Z. Anorg. Allg. Chem., 1969, 369, 327-340.

22 T. Nakajima, A. Mabuchi and R. Hagiwara, Carbon, 1988, 26, 357-361.

23 H. He, T. Riedl, A. Lerf and J. Klinowski, J. Phys. Chem., 1996, 100, 19954-19958.

24 W. Gao, L. B. Alemany, L. Ci and P. M. Ajayan, Nat. Chem., 2009, 1, 403-408.

25 W. Cai, R. D. Piner, F. J. Stadermann, S. Park, M. A. Shaibat, Y. Ishii, D. Yang, A. Velamakanni, S. J. An, M. Stoller, J. An, D. Chen and R. S. Ruoff, Science, 2008, 321, 1815-1817.

26 A. Bagri, C. Mattevi, M. Acik, Y. J. Chabal, M. Chhowalla and V. B. Shenoy, Nat. Chem., 2010, 2, 581-587.

27 S. Stankovich, D. A. Dikin, R. D. Piner, K. A. Kohlhaas, A. Kleinhammes, Y. Jia, Y. Wu, S. T. Nguyen and R. S. Ruoff, Carbon, 2007, 45, 1558-1565.

28 A. Ciesielski and P. Samorì, Chem. Soc. Rev., 2014, 43, 381398.

29 D. C. Marcano, D. V. Kosynkin, J. M. Berlin, A. Sinitskii, Z. Sun, A. Slesarev, L. B. Alemany, W. Lu and J. M. Tour, ACS Nano, 2010, 4, 4806-4814.

30 Z. Xu, M. Yue, L. Chen, B. Zhou, M. Shan, J. Niu, B. Li and X. Qian, Chem. Eng. J., 2014, 240, 187-194.

31 W. Gao, G. Wu, M. T. Janicke, D. A. Cullen, R. Mukundan, J. K. Baldwin, E. L. Brosha, C. Galande, P. M. Ajayan, K. L. More, A. M. Dattelbaum and P. Zelenay, Angew. Chem., Int. Ed. Engl., 2014, 53, 3588-3593.

32 B. Lesiak, L. Stobinski, A. Malolepszy, M. Mazurkiewicz, L. Kövér and J. Tóth, J. Electron Spectrosc. Relat. Phenom., 2014, 193, 92-99.

33 D. Ogrin, J. Chattopadhyay, A. K. Sadana, W. E. Billups and A. R. Barron, J. Am. Chem. Soc., 2006, 128, 11322-11323.

34 J. Chattopadhyay, A. Mukherjee, C. E. Hamilton, J. Kang, S. Chakraborty, W. Guo, K. F. Kelly, A. R. Barron and W. E. Billups, J. Am. Chem. Soc., 2008, 130, 5414-5415.

35 R. Nicholson, Anal. Chem., 1965, 37, 1351-1355.

36 C. H. A. Wong, A. Ambrosi and M. Pumera, Nanoscale, 2012, 4, 4972-4977.

37 H. L. Poh, F. Šaněk, A. Ambrosi, G. Zhao, Z. Sofer and M. Pumera, Nanoscale, 2012, 4, 3515-3522.

38 S. J. Konopka and B. McDuffie, Anal. Chem., 1970, 42, 17411746.

39 J. I. Paredes, S. Villar-Rodil, P. Solís-Fernández, A. MartínezAlonso and J. M. D. Tascón, Langmuir, 2009, 25, 5957-5968.

40 H. Estrade-szwarckopf, Carbon, 2004, 42, 1713-1721.

41 C. Hsieh, B. Yang and Y. Chen, Diamond Relat. Mater., 2012, 27-28, 68-75.

42 A. V. Ellis, A. Al-deen, H. Dalal and G. G. Andersson, J. Phys. Chem. C, 2013, 117, 21312-21319. 
43 Y. Sun, Z. Y. Wu, X. Wang, C. Ding, W. Cheng, S. H. Yu and X. Wang, Environ. Sci. Technol., 2016, 50, 4459-4467.

44 Y. Sun, C. Ding, W. Cheng and X. Wang, J. Hazard. Mater., 2014, 280, 399-408.

45 Y. Sun, X. Wang, W. Song, S. Lu, C. Chen and X. Wang, Environ. Sci.: Nano, 2017, 4, 222-232.

46 A. B. Dongil, B. Bachiller-Baeza, A. Guerrero-Ruiz and I. Rodríguez-Ramos, J. Catal., 2011, 282, 299-309.

47 Y. Sun, S. Yang, Y. Chen, C. Ding, W. Cheng and X. Wang, Environ. Sci. Technol., 2015, 49, 4255-4262.

48 J. L. Figueiredo, M. F. R. Pereira, M. M. A. Freitas and J. J. M. Orfao, Carbon, 1999, 37, 1379-1389.

49 J. L. Figueiredo, M. F. R. Pereira, M. M. a. Freitas and J. J. M. Órfão, Ind. Eng. Chem. Res., 2007, 46, 4110-4115.

50 A. B. Dongil, B. Bachiller-Baeza, A. Guerrero-Ruiz, I. Rodríguez-Ramos, A. Martínez-Alonso and J. M. D. Tascón, J. Colloid Interface Sci., 2011, 355, 179-189.

51 P. Solís-Fernández, R. Rozada, J. I. Paredes, S. Villar-Rodil, M. J. Fernández-Merino, L. Guardia, A. Martínez-Alonso and J. M. D. Tascón, J. Alloys Compd., 2012, 536, S532-S537.

52 S.-L. Kuo, W.-R. Liu, C.-P. Kuo, N.-L. Wu and H.-C. Wu, J. Power Sources, 2013, 244, 552-556.

53 A. Lerf, H. He, M. Forster and J. Klinowski, J. Phys. Chem. B, 1998, 5647, 4477-4482.

54 L. B. Casabianca, M. A. Shaibat, W. W. Cai, S. Park, R. Piner, R. S. Ruoff and Y. Ishii, J. Am. Chem. Soc., 2010, 5672-5676. 55 M. Acik, G. Lee, C. Mattevi, M. Chhowalla, K. Cho and Y. J. Chabal, Nat. Mater., 2010, 9, 840-845.

56 G. Lee, B. Lee, J. Kim and K. Cho, J. Phys. Chem. C, 2009, 113, 14225-14229.

57 F. A. Carey, Organic Chemistry, McGraw-Hill, University of Virginia, 4th edn, 2000.

58 Y. Sun, Q. Wang, C. Chen, X. Tan and X. Wang, Environ. Sci. Technol., 2012, 46, 6020-6027.

59 Y. Sun, S. Yang, C. Ding, Z. Jin and W. Cheng, RSC Adv., 2015, 5, 24886-24892.

60 Y. Sun, X. Wang, Y. Ai, Z. Yu, W. Huang, C. Chen, T. Hayat, A. Alsaedi and X. Wang, Chem. Eng. J., 2017, 310, 292-299.
$61 \mathrm{~J}$. Guerrero-Contreras and F. Caballero-Briones, Mater. Chem. Phys., 2015, 153, 209-220.

62 H. Kang, A. Kulkarni, S. Stankovich, R. S. Ruoff and S. Baik, Carbon, 2009, 47, 1520-1525.

63 M. S. Dresselhaus, A. Jorio, M. Hofmann, G. Dresselhaus and R. Saito, Nano Lett., 2010, 10, 751-758.

64 M. A. Pimenta, G. Dresselhaus, M. S. Dresselhaus, L. G. Cançado, A. Jorio and R. Saito, Phys. Chem. Chem. Phys., 2007, 9, 1276-1291.

65 A. C. Ferrari, J. C. Meyer, V. Scardaci, C. Casiraghi, M. Lazzeri, F. Mauri, S. Piscanec, D. Jiang, K. S. Novoselov, S. Roth and a. K. Geim, Phys. Rev. Lett., 2006, 97, 1-4.

66 D. Yang, A. Velamakanni, G. Bozoklu, S. Park, M. Stoller, R. D. Piner, S. Stankovich, I. Jung, D. A. Field, C. A. Ventrice and R. S. Ruoff, Carbon, 2009, 47, 145-152.

67 F. Lorestani, Z. Shahnavaz, P. Mn, Y. Alias and N. S. A. Manan, Sens. Actuators, B, 2015, 208, 389-398.

68 C. K. Chua and M. Pumera, Chemistry, 2014, 20, 1871-1877. 69 K. Krishnamoorthy, M. Veerapandian, K. Yun and S.-J. Kim, Carbon, 2013, 53, 38-49.

70 E. Fuente, J. A. Menéndez, M. A. Díez, D. Suárez and M. A. Montes-Morán, J. Phys. Chem. B, 2003, 107, 6350-6359.

71 G. Eda, J. Ball, C. Mattevi, M. Acik, L. Artiglia, G. Granozzi, Y. Chabal, T. D. Anthopoulos and M. Chhowalla, J. Mater. Chem., 2011, 21, 11217-11223.

72 V. H. Pham, T. V. Cuong, S. H. Hur, E. Oh, E. J. Kim, E. W. Shin and J. S. Chung, J. Mater. Chem., 2011, 21, 3371-3377.

73 Z. Luo, Y. Lu, L. A. Somers and A. T. C. Johnson, J. Am. Chem. Soc., 2009, 131, 898-899.

74 X. Ji, C. E. Banks, A. Crossley and R. G. Compton, ChemPhysChem, 2006, 7, 1337-1344.

75 A. Chou, T. Bocking, N. K. Singh and J. J. Gooding, Chem. Commun., 2005, 842-844.

76 S. M. Tan, A. Ambrosi, C. K. Chua and M. Pumera, J. Mater. Chem. A, 2014, 2, 10668-10675. 